\title{
287.
}

\section{NOTE ON THE EQUATION FOR THE SQUARED DIFFERENCES OF THE ROOTS OF A CUBIC EQUATION.}

[From the Quarterly Journal of Pure and Applied Mathematics, vol. III. (1860), pp. 307-309.]

THE question of finding the equation for the squared differences of the roots, presents, in the case of a cubic equation, a peculiarity which does not occur for equations of a higher order, viz. we may in the first instance form the equation for the differences of the roots taken in a given cyclical order, and thence deduce the equation for the squared differences of the roots. Let the cubic equation be

$$
U=\left(a, b, c, d \gamma(x, 1)^{3}=a(x-\alpha)(x-\beta)(x-\gamma)=0,\right.
$$

the function

$$
\Pi\{\theta-(\beta-\gamma)\},
$$

which equated to zero gives for $\theta$ the values $\beta-\gamma, \gamma-\alpha, \alpha-\beta$, which are the differences of the roots taken in a given circular ordər, has for any interchanges whatever of the roots, two values only, viz. that just written down, and the value $\Pi\{\theta-(\gamma-\beta)\}$, which may be deduced therefrom by changing first the sign of $\theta$ and then the sign of the entire expression (or what is the same thing, by changing the signs of the terms containing the even powers of $\theta$ ); we may consequently write

$$
\Pi\{\theta-(\beta-\gamma)\}=P-Q \zeta^{\frac{1}{2}}(\alpha, \beta, \gamma),
$$

which $P, Q$ are symmetrical functions of the roots, and $\zeta^{\frac{1}{2}}(\alpha, \beta, \gamma)$ or $(\alpha-\beta)(\beta-\gamma)(\gamma-\alpha)$ is a function the square of which is a symmetrical function of the roots, and such 
symmetrical functions of the roots can of course be expressed as functions of the coefficients. We have in fact

$$
\begin{aligned}
& P=\theta^{3}+\theta\left(\Sigma \beta \gamma-\Sigma \alpha^{2}\right)=a^{-2}\left\{a^{2} \theta^{3}+9\left(a c-b^{2}\right) \theta\right\} \\
& Q=1
\end{aligned}
$$

and

$$
\zeta^{\frac{1}{2}}(\alpha, \beta, \gamma)=a^{-2} \sqrt{ }-27 \square,
$$

where $\square$ is the discriminant of the cubic function,

$$
=a^{2} d^{2}-6 a b c d+4 a c^{3}+4 b^{3} d-3 b^{2} c^{2} .
$$

Consequently

$$
\Pi\{\theta-(\beta-\gamma)\}=a^{-2}\left\{a^{2} \theta^{3}+9\left(a c-b^{2}\right) \theta-\sqrt{-27 \square}\right\},
$$

and forming the similar equation

$$
\Pi\{\theta+(\beta-\gamma)\}=a^{-2}\left\{a^{2} \theta^{3}+9\left(a c-b^{2}\right) \theta+\sqrt{-27 \square}\right\},
$$

multiplying the two equations together and writing $u$ in the place of $\theta^{2}$, we find

$$
\Pi\left\{u-(\beta-\gamma)^{2}\right\}=a^{-4}\left\{\left[a^{2} u+9\left(a c-b^{2}\right)\right]^{2} u+27 \square\right\},
$$

and the equation for the squared differences of the roots is thus seen to be

$$
\left[a^{2} u+9\left(a c-b^{2}\right)\right]^{2} u+27 \square=0,
$$

or what is the same thing

$$
a^{4} u^{3}+18 a^{2}\left(a c-b^{2}\right) u^{2}+81\left(a c-b^{2}\right)^{2} u+27 \square=0 .
$$

I remark that if $\omega$ is an imaginary cube root of unity (so that $\left(\omega-\omega^{2}\right)^{2}=-3, \omega-\omega^{2}$ being thus only another form of $\sqrt{-3})$ then if in the expression for $\Pi\{\theta-(\beta-\gamma)\}$ we write $\frac{3 \theta}{\left(\omega-\omega^{2}\right) a}$ in the place of $\theta$, the equation assumes the more simple form

$$
\Pi\left\{\theta-\frac{1}{3} a\left(\omega-\omega^{2}\right)(\beta-\gamma)\right\}=\theta^{3}-3\left(a c-b^{2}\right) \theta-a \sqrt{\square},
$$

which if $U$ be the cubic function, $H$ the Hessian $=\left(a c-b^{2}, a d-b c, b d-c^{2}\right)(x, y)^{2}$, and $\square$ the discriminant as before, is a particular case (obtained by writing $x=1, y=0$ ) of the equation

$$
\Pi\left\{\theta-\frac{1}{3} a\left(\omega-\omega^{2}\right)(x-\alpha y)\right\}=\theta^{3}-3 H \theta-U \sqrt{\square},
$$

which equation can be at once obtained from the equation (where $\Phi$ is the cubicovariant of the cubic function)

$$
\sqrt[3]{\frac{1}{2}(\Phi+U \sqrt{\square})}-\sqrt[3]{\frac{1}{2}(\phi-U \sqrt{\square})}=\frac{1}{3} a\left(\omega-\omega^{2}\right)(\beta-\gamma)(x-\alpha y)
$$


given in my Fifth Memoir on Quantics, Phil. Trans., t. cxlviII. (1858), [156]. For writing for a moment

$$
\theta=\sqrt[3]{\bar{X}}-\sqrt[3]{\bar{Y}}
$$

we find

$$
\theta^{3}=X-Y-3 \sqrt[3]{X Y \theta}
$$

or

$$
\theta^{3}+3 \sqrt[3]{X Y \theta}-(X-Y)=0
$$

where $\sqrt[3]{X Y}=\sqrt[3]{\frac{1}{4}\left(\Phi^{2}-U^{2} \square\right)}$, which by the equation

$$
\phi^{2}-U^{2} \square=-4 H^{3}
$$

(given in the Memoir) is $=-H$, and $(X-Y)$ is $=U \sqrt{\square}$, so that the equation in $\theta$ is, as above, $\theta^{3}-3 H \theta-U \sqrt{ } \square=0$, an equation which is satisfied by $\theta=\frac{1}{3} a\left(\omega-\omega^{2}\right)(\beta-\gamma)(x-\alpha y)$; and the other two roots being of course of the like form, the cubic function in $\theta$ is equal to $\Pi\left\{\theta-\frac{1}{3} a\left(\omega-\omega^{2}\right)(\beta-\gamma)(x-\alpha y)\right\}$ which proves the theorem.

2, Stone Buildings, W.C., Nov. 3rd, 1859.

C. IV. 